Muro de la Investigación, 2021(1), enero-junio

ISSN: 2523-2886

Doi: https://doi.org/10.17162/rmi.v6i1.1437

\title{
Adicción a redes sociales y ciberbullying en los adolescentes
}

\author{
Addiction to social networks and cyberbullying in adolescents \\ Doris Chiza-Lozano ${ }^{1}$ Devora Vásquez-Mendoza ${ }^{2 a}$ y Celina Ramírez Vega ${ }^{3}$ \\ Universidad Peruana Unión, Perú ${ }^{1,2,3}$
}

Recibido: 15 de agosto de 2020

Aceptado: 12 de diciembre de 2020

\begin{abstract}
Resumen
El objetivo del estudio fue determinar si existe relación significativa entre adicción a redes sociales y ciberbullying en los adolescentes que estudian en la Asociación Educativa Adventista Andina Central. Sigue los lineamientos de un diseño no experimental de corte transversal y de tipo correlacional. Participaron del estudio 326 adolescentes del nivel secundario de ambos sexos entre 12 y 17 años de edad. Para la medición de las variables, se utilizaron; el Cuestionario de Adicción a Redes Sociales (ARS) construida por Escurra y Salas (2014) y el European Ciberbullying Intervention Project Questionnaire (ECIPQ), ambos instrumentos son válidos y confiables de acuerdo a los mismos. Por otro lado, los datos fueron procesados, realizándose análisis descriptivos y de correlación. Se encontró que la adicción a redes sociales y el ciberbullying se relacionan significativamente $\left(\mathrm{r}=.381^{*}\right.$, $\mathrm{p}$ $=0.00)$. Es decir, los participantes que tienen un mayor nivel de adicción a redes sociales a su vez presentan mayores indicadores de desarrollar ciberbullying.
\end{abstract}

Palabras clave: Adicción a redes sociales, ciberbullying, adolescentes, escolares, secundaria.

\begin{abstract}
The objective of the study was to determine if there is a significant relationship between addiction to social networks and cyberbullying in adolescents who study at the Central Andean Adventist Educational Association. It follows the guidelines of a non-experimental, cross-sectional, correlational design. 326 adolescents from the secondary level of both sexes between 12 and 17 years of age participated in the study. For the measurement of the variables, they were used; the Social Media Addiction Questionnaire (ARS) constructed by Escurra and Salas (2014) and the European Cyberbullying Intervention Project Questionnaire (ECIPQ), both instruments are valid and reliable according to them. On the other hand, the data were processed, performing descriptive and correlation analyzes. It was found that addiction to social networks and cyberbullying are significantly related $(\mathrm{r}=.381 * *, \mathrm{p}=$ 0.00). That is, the participants who have a higher level of addiction to social networks in turn present higher indicators of developing cyberbullying.
\end{abstract}

${ }^{\mathrm{a}}$ Correspondencia al autor:

E-mail: devoravasquez@upeu.edu.pe 
Keywords: Addiction to social networks, cyberbullying, adolescents, schoolchildren, high school

\section{Introducción}

Actualmente, el uso de internet se ha convertido en la normalidad de la persona, pues le permite trabajar, estudiar, relajarse, entre otras actividades. En este sentido, internet se ha convertido en una herramienta importante para la comunicación (Sapountzi \& Psannis, 2018). Las redes sociales están proporcionando nuevos esquemas y recursos para la interacción social en un entorno de comunicación (Pivec \& Macek, 2019), los cuales han reemplazado gradualmente a la comunicación personal, accediendo a una comunicación rápida y fluida sin los límites de la distancia o el tiempo (Hechanova \& Ortega, 2014).

A nivel mundial, casi 1,900.00 millones de personas utilizan Facebook; 1,200 millones usan WhatsApp; alrededor de 1,000 millones consumen videos de YouTube; otras 600 millones de personas usan Instagram y 319 millones Twitter; por otro lado, estas redes sociales están generando un riesgo de adicción muy alto, lo que puede provocar alteraciones en su salud emocional y/o mental (Bedir, 2016).

Los especialistas conceptúan a la adicción como el conjunto de un patrón de conductas potencialmente riesgosas y puede incluir un consumo de sustancias perjudiciales para el consumidor (Echeburúa \& De Corral, 2010). Por ende, Kuss y Griffiths (2011) lo consideraron un problema de conducta (Campos \& Vilchez, 2019; Peña et al., 2019; Valencia-Ortiz \& Cabero-Almenara, 2019)

A partir de un ángulo organizacional, se afirma que la adhesión excesiva a las redes sociales puede convertirse en una preocupación entre los jóvenes. Por ejemplo, en una encuesta a 120 administradores y profesionales de labor juvenil, Davis, Flett y Besser (2002) informaron que sus participantes temían que el uso de las redes sociales desplazara otras actividades y la interacción social personal y que ello conlleve a conductas como el acoso cibernético.

Por ello, el ciberbullying consiste en usar las Tecnologías de Información y Comunicación (TICs) mediante internet para ejercer acoso entre iguales (Garaigordobil, 2017). Según otra investigación, el $20.4 \%$ de adolescentes manifiestan ser víctimas de insultos u otro tipo de violencia (Martínez-Ferrer \& Moreno Ruiz, 2017). En el Perú, de los 
11,369 casos reportados por violencia escolar, 440 registros provienen de ciberbullying. Ante esta situación, en el 2019 se publicaron 577 artículos relacionados a ciberbullying (Scopus, 2020). El trabajo más consultado es el de Smith et. al. (2008), que analiza el impacto y la naturaleza del ciberbullying en estudiantes de nivel secundario.

Estudios anteriores muestran la relación entre acoso cibernético y uso de redes sociales, y que los perpetradores cibernéticos obtuvieron resultados altos en relación al uso inseguro de redes sociales (Griffiths et al., 2014). Por su parte, Shadmanfaat et. al. (2020) explica que el ciberacoso se muestra en el contexto donde las personas adquieren respuestas agresivas de la misma manera que adquieran otras formas de aprendizaje, sea por experiencia directa o por observación; mientras que Sierra-Arévalo y Papachristos (2017) afirman que las características y rasgos de personalidad de las personas pueden convertirse en posibles predictores de una conducta agresiva (Bouquet-Escobedo, et al., 2019).

Desde otro aspecto, Escurra y Salas (2014) conceptúan la adicción a las redes sociales en base a tres elementos que forman un grupo de síntomas: el factor obsesivo a las redes sociales (síntoma cognitivo, fantaseo con las redes sociales); el factor de descontrol personal, (síntoma conductual con descuido de tareas); y el factor excesivo de uso de redes sociales (síntoma funcional de dependencia extrema de las redes sociales).

En este sentido, el objetivo de la presente investigación es determinar si existe alguna relación significativa entre la adicción a las redes sociales y el ciberbullying en los adolescentes de la Asociación Educativa Adventista Andina Central, 2020.

\section{Metodología de la investigación}

Este estudio sigue un enfoque cuantitativo, de diseño no experimental y corte transversal, puesto que no habrá manipulación de las variables y los datos serán recogidos en solo momento. También, es de tipo correlacional, pues permite analizar la relación entre las variables del estudio (Hernández, Fernández \& Baptista, 2014): adicción a las redes sociales y ciberbullying.

La población de estudio consiste en 400 alumnos, de los que participaron 326 adolescentes de ambos sexos de todo el nivel secundario de la Asociación Educativa Adventista Andina Central, cuyas edades fluctúan entre 12 y 17 años ( $M=14.23 ; \mathrm{DS}=1.482)$. Asimismo, se excluyó a adolescentes menores de 12 años y mayores de 17, también a 
adolescentes que omitan más del $10 \%$ de preguntas en los instrumentos de medición y que no cuenten con el respectivo consentimiento informado.

Se utilizó el cuestionario ARS (Escurra y Salas, 2014), el cual evalúa la adicción a redes sociales, y está compuesta por 24 ítems con cinco opciones de respuesta: nunca, rara vez, algunas veces, casi siempre y siempre. De acuerdo a Escurra y Salas (2014), el ARS es válido $(\mathrm{GFI}=.92 ; \mathrm{RMR}=.06 ; \mathrm{RMSEA}=.04 ; \mathrm{AIC}=477.28)$ y confiable $(\alpha=.88)$; y la confiabilidad del mismo en el presente estudio fue buena $(\alpha=.94)$, y para la dimensión obsesión por las redes sociales $(\alpha=.85)$, falta de control personal en el uso de las redes sociales $(\alpha=.82)$ y uso excesivo de las redes sociales $(\alpha=.90)$.

Por otro lado, el European Cyberbullying Intervention Project Questionnaire (ECIPQ), diseñado Del Rey et al. (2015) se compone de 22 ítems distribuidos en 2 dimensiones: cibervictimización y ciberagresión, con cinco opciones de respuesta de tipo Likert donde 0 es nunca y 4 siempre. Dichos autores sostienen que el ECIPQ es válido (CFI $=.96, \mathrm{IFI}=.96, \mathrm{RMSEA}=.05)$ y confiable $(\alpha=.87)$. Respecto a la confiabilidad del ECIPQ, en la presente investigación, fue $\alpha=.82$ siendo aceptable; de la misma manera, se obtuvo índices de confiabilidad satisfactorios para la dimensión cibervictimización $(\alpha=.80)$ y ciberagresión $(\alpha=.81)$.

La recolección de la información se realizó colectivamente en formato digital, y los cuestionarios se elaboraron a través de la plataforma Google Forms. A través de un formulario virtual, se explicó los fines del estudio y se enfatizó que la participación es de carácter voluntario y confidencial.

Finalmente, la recolección de la información dio lugar a utilizar la base de datos en el software estadístico SPSS para Windows versión 24. Posteriormente, se verificó los datos a fin de evitar valores perdidos y atípicos (limpieza de datos), y se ejecutaron los análisis descriptivos e inferenciales previo análisis de la distribución muestral.

\section{Resultados}




\section{Descripción demográfica de los participantes}

La Tabla 1, revela las características demográficas de los estudiantes de los cuales $48.5 \%$ son masculinos y $51.5 \%$ de sexo femenino; además que $16.3 \%$ de ellos son de 12 años, $18.4 \%$ de 13 años, $19.3 \%$ de 14 años, $23 \%$ de 15 años, $17.8 \%$ de 16 y $5.2 \%$ estudiantes tienen 17 años; mencionando a la cantidad de alumnos por año de estudios $18.1 \%$ son de primer año, $16.6 \%$ de segundo, $21.2 \%$ de tercero, $23 \%$ de cuarto y $21.2 \%$ de quinto año.

Tabla 1

Descripción demográfica de los participantes

\begin{tabular}{lccc}
\hline & & Frecuencia & Porcentaje \\
\hline Sexo & Masculino & 158 & 48.5 \\
& Femenino & 168 & 51.5 \\
& & & \\
\multirow{5}{*}{ Edad } & 12 & 53 & 16.3 \\
& 13 & 60 & 18.4 \\
& 14 & 63 & 19.3 \\
& 15 & 75 & 17.0 \\
& 16 & 58 & 5.2 \\
Año de estudios & 17 & 59 & 18.1 \\
& & 54 & 16.6 \\
& Primero & 69 & 21.2 \\
& Segundo & 75 & 23.0 \\
& Tercero & 69 & 21.2 \\
& Cuarto & 326 & 100.0 \\
\hline
\end{tabular}

\section{Niveles de adicción a las redes sociales y sus dimensiones}

Se observa en la Tabla 2 que el 35.3\% de los adolescentes de la Asociación Educativa Adventista Andina Central, presentan un nivel promedio de adicción a redes sociales. Respecto a la dimensión obsesión por las redes sociales un 36.5\% de adolescentes se ubican en este nivel; en falta de control personal en el uso de las redes sociales $36,2 \%$ y uso excesivo de las redes sociales también $36,2 \%$.

\section{Tabla 2}

Niveles de adicción a redes sociales de los adolescentes de la Asociación Educativa Adventista Andina Central 


\begin{tabular}{|c|c|c|c|c|c|c|}
\hline \multirow{2}{*}{ Variables } & \multicolumn{2}{|c|}{ Bajo } & \multicolumn{2}{|c|}{ Promedio } & \multicolumn{2}{|c|}{ Alto } \\
\hline & $\mathrm{f}$ & $\%$ & $\mathrm{f}$ & $\%$ & $\mathrm{f}$ & $\%$ \\
\hline Adicción a las redes sociales & 97 & $29.8 \%$ & 115 & $35.3 \%$ & 114 & $35.0 \%$ \\
\hline $\begin{array}{l}\text { Obsesión por las redes } \\
\text { sociales. }\end{array}$ & 98 & $30.1 \%$ & 109 & $33.4 \%$ & 119 & $36.5 \%$ \\
\hline $\begin{array}{l}\text { Falta de control personal en el } \\
\text { uso de las redes sociales. }\end{array}$ & 93 & $28.5 \%$ & 115 & $35.3 \%$ & 118 & $36.2 \%$ \\
\hline $\begin{array}{l}\text { Uso excesivo de las redes } \\
\text { sociales. }\end{array}$ & 99 & $30.4 \%$ & 109 & $33.4 \%$ & 118 & $36.2 \%$ \\
\hline
\end{tabular}

\section{Niveles de ciberbullying y sus dimensiones}

En la Tabla 3, se aprecia que el $46.6 \%$ de los adolescentes presentan un nivel bajo de ciberbullying, el $34.7 \%$ un nivel alto y un $18.7 \%$ un nivel promedio. Respecto a la dimensión cibervictimización el $62.3 \%$ se ubica en un nivel bajo, de manera similar ocurre en la dimensión ciberagresión (71.5\%).

\section{Tabla 3}

Niveles de ciberbullying de los adolescentes de la Asociación Educativa Adventista Andina Central

\begin{tabular}{ccccccc}
\hline \multirow{2}{*}{ Variables } & \multicolumn{2}{c}{ Bajo } & \multicolumn{2}{c}{ Promedio } & \multicolumn{2}{c}{ Alto } \\
\cline { 2 - 7 } & $\mathrm{f}$ & $\%$ & $\mathrm{f}$ & $\%$ & $\mathrm{f}$ & $\%$ \\
\hline Ciberbullying & 152 & $46.6 \%$ & 61 & $18.7 \%$ & 113 & $34.7 \%$ \\
Cibervictimización & 203 & $62.3 \%$ & 35 & $10.7 \%$ & 88 & $27.0 \%$ \\
Ciberagresión & 233 & $71.5 \%$ & 36 & $11.0 \%$ & 57 & $17.5 \%$ \\
\hline
\end{tabular}

\section{Prueba de normalidad}

En la Tabla 3, se aprecia que la prueba de bondad de ajuste de Kolmogorov-Smirnov (K-S), muestra que los datos siguen una distribución asimétrica por lo que se utilizará la estadística no paramétrica.

\section{Tabla 4}

Prueba de bondad de ajuste a la curva normal para las variables de estudio

\begin{tabular}{ccccc}
\hline Variable & Media & D.E. & K-S & p \\
\hline Adicción a redes sociales & 56,52 & 16,890 &, 106 & 0,000 \\
& & & & \\
\hline
\end{tabular}




\begin{tabular}{|c|c|c|c|c|}
\hline Obsesión por las redes sociales & 22,92 & 6,891 & 130 & 0,000 \\
\hline Falta de control en el uso de las redes sociales & 14,09 & 4,691 & 102 & 0,000 \\
\hline Uso excesivo de las redes sociales & 19,51 & 6,378 & ,077 & 0,000 \\
\hline Ciberbullying & 3,56 & 4,885 & ,233 & 0,000 \\
\hline
\end{tabular}

\section{Análisis de correlación}

En la Tabla 5, el coeficiente de correlación rho de Spearman indica que existe una relación directa y altamente significativa entre adicción a redes sociales y ciberbullying (rho $=.381, \mathrm{p}=0.00)$. De manera similar ocurre entre las demás dimensiones de la ARS y el ciberbullying.

\section{Tabla 5}

Coeficientes de correlación entre adicción a redes sociales y ciberbullying

\begin{tabular}{lcc}
\hline Adicción a redes sociales y sus dimensiones & \multicolumn{2}{c}{ Ciberbullying } \\
\hline & rho & $\mathrm{p}$ \\
\cline { 2 - 3 } Adicción a redes sociales & $.381^{* *}$ & 0.000 \\
Obsesión por las redes sociales & $.264^{* *}$ & 0.000 \\
Falta de control en el uso de las redes sociales & $.362^{* *}$ & 0.000 \\
Uso excesivo de las redes sociales & $.450^{* *}$ & 0.000
\end{tabular}

** La correlación es significante a nivel 0.01

* La correlación es significante a nivel 0.05

\section{Discusión}

El objetivo de este trabajo fue determinar la relación significativa entre "adicción a redes sociales" y "ciberbullying" en los adolescentes de la Asociación Educativa Adventista Andina Central, 2020. Se encontró que existe una relación directa y altamente significativa entre la variables de estudio ( $r h o=.381 ; \mathrm{p}<.00)$; significando que los participantes que presentan un nivel elevado de adicción a redes sociales, a su vez exhiben mayores 
propensiones de realizar ciberbullying (Griffiths, Kuss \& Demetrovics, 2014). Por consiguiente, las carencias que sufre un ser humano a nivel social afectan su relación con otros y potencian el maltrato, tal como lo afirma el modelo de Fontaine y Dodge (2006).

Por su parte, Turel y Serenko (2012) destacan que los factores desestabilizantes de maltrato pueden ser ocasionados por el entorno adictivo de una persona. Además, el uso compulsivo de redes sociales, muestran una relación directo con el ciberbullying (Griffiths et al., 2014; Cantero, 2016; Smith et al., 2008).

En cuanto a la dimensión "obsesión por las redes sociales," esta posee una relación significativa (rho=.264; $\mathrm{p}<.00)$ con la variable "ciberbullying;" que significa que las personas que presentan mayor nivel de obsesión por estar conectados con redes sociales, presentan elevadas posibilidades de padecer u practicar ciberbullying. En este sentido, Pivec y Macek (2019) reiteran que las dificultades de las personas para comunicarse conducen a desconectarse del mundo real y pasar a la esfera virtual, en la cual aumenta la posibilidad de sufrir o de ser un agresor. En este caso, Hechanova y Ortega (2014) afirman que los lazos débiles en entornos familiares o amicales son características generales de los abusadores, con un contexto familiar de violencia o agresión (Milner y Robertson, 1990), destacando el papel importante del clima familiar en la predicción de conductas de agresión.

La dimensión "falta de control en el uso de las redes sociales" reveló una relación directa significativa con "ciberbullying" (rho=.362; $\mathrm{p}<.00)$; esto significa que, a mayor falta de control en el uso en las redes sociales, mayor es el riesgo de experimentar maltrato en un entorno virtual. En este sentido, la falta de control en el uso de las redes sociales provoca anomalías diversas en los usuarios, y estos, mientras menos control tengan, tendrán más probabilidades de caer en conductas de maltrato (Cantero, 2016); esto resulta en que, en promedio, $42 \%$ de los niños son víctimas de ciberacoso (Chisholm, 2006).

Por su parte, se observa una relación significativa evidente entre la dimensión "uso excesivo de las redes sociales" y "ciberbullying" (rho=.450; $\mathrm{p}<.00)$; lo que significa: al darse situaciones en las que se hace patente el uso excesivo de las redes sociales, el ciberbullying crece de manera continua. Algunas estadísticas afirman que cada año 1 billón de niños experimentan algún tipo de violencia; ante esto, Gelle (1980) afirma la aparición del ciclo de violencia como un factor preponderante en el maltrato, sea virtual o real. 
Finalmente, en algunos contextos similares, se observa cómo las adicciones provocan una influencia directa en la aparición de violencia, ya sea ésta aumentando la cantidad de víctimas o aumentando la cantidad de perpetradores. Por ende, es importante destacar la presencia de posibles indicadores relacionados a la adicción a redes sociales como un potencial de conducta violenta. Más aún, porque son los adolescentes y niños los que no tienen la capacidad adecuada de control que sí puede experimentar el adulto. Además, la presencia de un entorno de hogar adecuado, se convierte en un componente ideal para evitar situaciones de agresión o de bullying, de cualquier clase.

\section{Referencias}

Bedir, H. (2016). The perspectives of young adolescent EFL learners on critical thinking skills. International Online Journal of Education and Teaching, 3(3), 229-238. Retrieved from http://iojet.org/index.php/IOJET/article/view/145/136

Bouquet-Escobedo, G. S., García-Mendez, M., Díaz-Loving, R., \& Rivera-Aragon, S. (2019). Conceptuación y medición de la agresividad: validación de una escala. Revista Colombiana de Psicología, 28(1), 115-130.

Cantero, C. (2016). Una revisión: Ciberacoso "Entre Iguales", otra demanda social. Escenarios, 1(19), 58-66. doi:10.21892/20119097.299

Cyberbulling: acoso que no frena en la región Junín. (17 de agosto de 2018). Diario el Correo Edición Huancayo, Recuperado de https://diariocorreo.pe/edicion/huancayo/cyberbullying-violencia-que-se-comparte$836425 /$ ?ref $=\mathrm{dcr}$

Chisholm, J. F. (2006). Cyberspace Violence against Girls and Adolescent Females. Annals of the New York Academy of Sciences, 1087(1), 74-89. doi:10.1196/annals.1385.022

Crick, N. y Dodge, K. (1994). A review and reformulation of social information-processing mechanisms in children's social adjustment. Psychological Bulletin, 115, 74-101. doi:10.1037/0033- 2909.115.1.74

Colwell, J., \& Kato, M. (2003). Investigation of the relationship between social isolation, self-esteem, aggression and computer game play in Japanese adolescents. Asian Journal of Social Psychology, 6(2), 149-158. doi:10.1111/1467-839X.t01-1-00017

Davis, R. A., Flett, G. L., \& Besser, A. (2002). Validation of a New Scale for Measuring Problematic Internet Use: Implications for Pre-employment Screening. CyberPsychology \& Behavior, 5(4), 331-345. doi:10.1089/109493102760275581

Dooley, J. J., Pyzalski, J., \& Cross, D. (2009). Cyberbullying versus face-to-face bullying: A theoretical and conceptual review. Journal of Psychology, 217(4), 182-188. doi:10.1027/0044-3409.217.4.182

Del Rey, R., Casas, J.A., Ortega-Ruiz, R., Schultze-Krumbholz, A., Scheithauer, H., Smith, P. Plichta, P. (2015). Structural validation and cross-cultural robustness of the 
European Cyberbullying Intervention Project Questionnaire. Computers in Human Behavior, 50, 141-147. doi:10.1016/j.chb.2015.03.065.

Echeburúa, E., \& De Corral, P. (2010). Adicción a las nuevas tecnologías y a las redes sociales en jóvenes: un nuevo reto. Adicciones, 22(2), 91-96. doi: 10.20882/adicciones. 196

Escurra, M. y Salas, E. (2014). Construcción y validación del cuestionario de Adicción a Redes Sociales (ARS). Liberabit, 20(1), 73-91. Recuperado de http://www.redalyc.org/articulo.oa?id=68631260007

Fontaine, R.G. and Dodge, K.A. (2006), Real-time decision making and aggressive behavior in youth: a heuristic model of response evaluation and decision (RED). Aggr. Behav., 32,604-624. doi:10.1002/ab.20150

Garaigordobil, M. (2015). Cyberbullying en adolescentes y jóvenes del País Vasco: Cambios con la edad. Anales de Psicología, 31(3), 1069. doi:10.6018/analesps.31.3.179151

Garaigordobil, M. (2017). Conducta antisocial: conexión con bullying/cyberbullying y estrategias de resolución de conflictos. Psychosocial Intervention, 26(1), 47-54. doi: 10.1016/j.psi.2015.12.002

Griffiths, M. D., Kuss, D. J., \& Demetrovics, Z. (2014). Social Networking Addiction: An Overview of Preliminary Findings. In K. Rosenberg \& L. Curtiss (Eds.), Behavioral Addictions: Criteria, Evidence, and Treatment (119-141). doi: 10.1016/B978-0-12407724-9.00006-9

Gelles, R. J. (1980). Violence in the family: A review of research in the seventies. Journal of Marriage and the Family, 42(4), 873-885. doi:10.2307/351830

Hechanova, R., \& Ortega-Go, R. (2014). The Good, the Bad and the Ugly: Internet Use, Outcomes and the Role of Regulation in the Philippines. The Electronic Journal of Information Systems in Developing Countries, 63(8),1-25. doi: 10.1002/j.16814835.2014.tb00453.

Hernández, R., Fernández, C., \& Baptista, P. (2014). Metodología de la investigación. México D.F.: McGraw-Hill.

Karina, P. (11 de setiembre de 2020). 33\% de la generación $\mathrm{Z}$ ha tenido que cerrar sus redes sociales por cyberbullying. Publimetro. https://www.publimetro.cl/cl/noticias/2020/09/11/cyberbullying.html

Kernberg, O (2,005). Agresividad, Narcisismo $Y$ autodestrucción en la relación psicoterapéutica. Barcelona: Editorial Paidós Ibérica. 
Kircaburun, K., Demetrovics, Z., Király, O., \& Griffiths, M. D. (2020). Childhood emotional trauma and cyberbullying perpetration among emerging adults: A multiple mediation model of the role of problematic social media use and psychopathology. International Journal of Mental Health and Addiction, 18(3), 548-566. doi:10.1007/s11469-0189941-5

Kuss, D. J., \& Griffiths, M. D. (2011). Online social networking and addiction-A review of the psychological literature. International Journal of Environment Research and Public Health, 8(9), 3528- 3552. doi:10.3390/ijerph8093528

Martínez-Ferrer, B., \& Moreno Ruiz, D. (2017). Dependencia de las redes sociales virtuales y violencia escolar en adolescentes. International Journal of Developmental and Educational Psychology, 2(1), 105-114. doi: 10.17060/ijodaep.2017.n1.v2.923

Milner, J.S., Robertson, K.R., y Rogers, D.L. (1990). Childhood history of abuse and adult child abuse potential. Journal of Family Violence, 5, 15-34. Recuperado de: https://psycnet.apa.org/record/1990-25603-001

Peña, G., Ley, S., Castro, J., Madrid, P., Apodaca, F., \& Aceves, E. (2019). Predominio de las TIC y adicción a las redes sociales en estudiantes universitarios del área de salud. RITI Journal, 7(13), 83-91. Recuperado de: https://www.riti.es/ojs2018/inicio/index.php/riti/article/view/157

Pivec, M., \& Macek, A. (2019). Employment background influence on social media usage in the field of European project management and communication. Journal of Business Research, 94, 280-289. doi: 10.1016/j.jbusres.2018.03.021

Sapountzi, A., \& Psannis, K. (2018). Social networking data analysis tools \& challenges. Future Generation Computer Systems, 86, 893-913. doi: 10.1016/j.future.2016.10.019

Shadmanfaat, S. M., Howell, C. J., Muniz, C. N., Cochran, J. K., Kabiri, S., \& Fontaine, E. M. (2020). Cyberbullying perpetration: An empirical test of social learning theory in iran. Deviant Behavior, 41(3), 278-293. doi:10.1080/01639625.2019.1565513

Sierra-Arévalo, M., \& Papachristos, A. V. (2017). Social Networks and Gang Violence Reduction. Annual Review in Advance, 19, 1-21. doi: 10.1146/annurev-lawsocsci110615-085017

Smith, P. K., Mahdavi, J., Carvalho, M., Fisher, S., Russell, S., \& Tippett, N. (2008). Cyberbullying: Its nature and impact in secondary school pupils. Journal of Child Psychology and Psychiatry and Allied Disciplines, 49(4), 376-385. https://doi.org/10.1111/j.1469-7610.2007.01846.x

Turel, O., \& Serenko, A. (2012). The benefits and dangers of enjoyment with social networking websites. European Journal of Information Systems, 21(5), 512-528. doi:10.1057/ejis.2012.1

Valencia-Ortiz, R., \& Cabero-Almenara, J. (2019). La adicción a las redes sociales: validación de un instrumento en el contexto mexicano. Health and Addictions, 19(2), 149-159. Recuperado de: https://ojs.haaj.org/index.php/haaj/article/view/460 